\title{
Robot-Control Method Based on Personal Space
}

\author{
Kota Kawamoto, Ryusuke Fujisawa, Eiji Hayashi \\ Department of Mechanical Information Science and Technology, Kyushu Institute of Technology \\ 680-4, Kawazu, Iizuka-City, Fukuoka, 820-8502, Japan
}

E-mail:kawamoto@mmcs.mse.kyutech.ac.jp,fujisawa@.ces.kyutech.ac.jp, haya@.mse.kyutech.ac.jp

\begin{abstract}
In our daily life, we unconsciously adjust our personal space according to the intimacy with others. It is also said that personal space is classified into non-verbal communication. In this research, we analyzed the distance that a person can tolerate when the robot approaches to identify the minimum distance that the person is able to interact comfortably. As a result, we could get parameters which can be index of initial distance between robot and users.
\end{abstract}

Keywords: personal robot, personal space, nonverbal communication, intimacy

\section{Introduction}

Nowadays, the rapid development of high technology has produced robots not only for industrial factories (industrial robots) but also for museums, homes, healthcare institutions and so on (non-industrial robots). Among various types of non-industrial robots, recently, service and personal robots have attracted global attention[1]. What makes them unique is that they are required to have "user affinity" that gives the user a sense of affinity as well as functions such as intellectual behavior and communication ability. Generally, human communication can be divided into two simple categories verbal communication and nonverbal communication. Moreover, it is said that almost 60 percent of the human communication consists of nonverbal communication whether through facial expression, the tone of voice or body movement[2]. All of these are essential to conveying intent or emotional state. Thus, robot control system should be considered the impression from the standpoint of users.

E.T. Hall introduced for the first time the concept of interpersonal distances[3]. People create their own territories around them unconsciously, which define and determine the interactions they can have with other people. In a word, we all are creating our own portable bubble in public space to keep others far from each other. Interpersonal distances are classified into non-verbal communication. Furthermore, the interpersonal distance between them is reflected the social relationship they have. E.T. Hall has proposed a model to represent these different spaces; it divides the space around a person in 4 distinct zones (i), (ii), (iii), (iv), as shown in Figure 1.

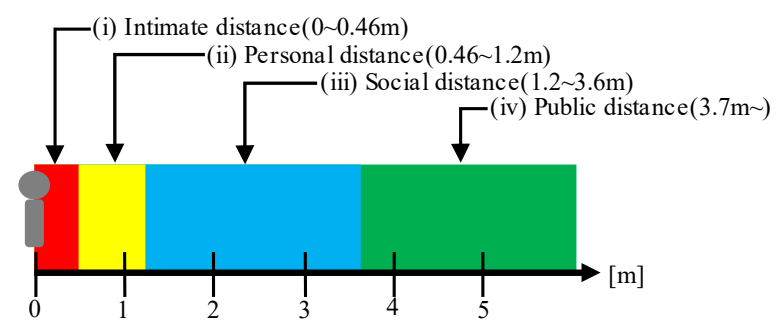

Fig. 1. Definition of Hall's personal space model. 
He defined that the Intimate distance is for embracing, touching or whispering, the Personal distance is for interactions among good friends or family, the Social distance is for interaction among acquaintances, the Public distances used for public speaking. Moreover, space within (i) intimate distance and (ii) personal distance is called personal space.

In this research, we designed the robot system and conducted an experiment with considering the personal space between human and robots.

\section{Structure of the Robot}

The robot we developed is depicted in Figure 2. This robot consists of 4 major parts: the head with 2 degrees of freedom, the Body with 3 degrees of freedom, the Arm with 6 degrees of freedom and the Base with a 4-wheel-drive. The height of the robot is approximately $1.5 \mathrm{~m}$

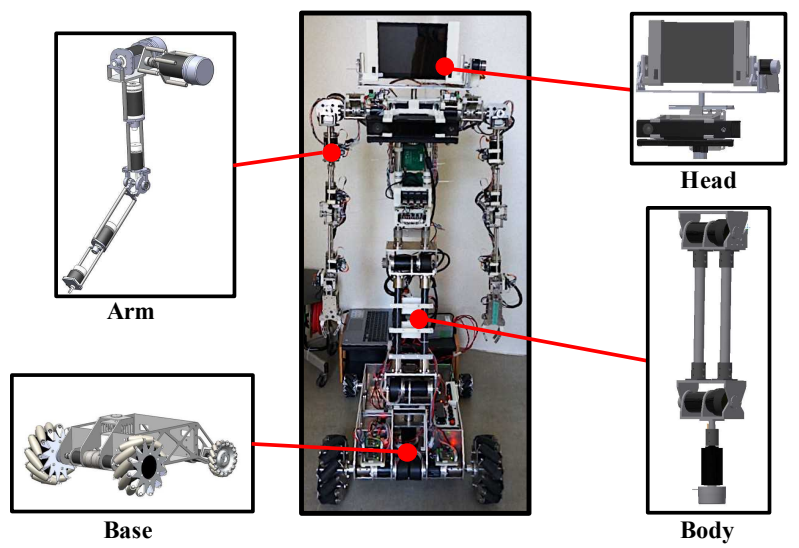

Fig. 2. Structure of the robot

\section{System Configuration}

The control diagram is depicted in Figure 3. In this system, each part of the robot constructs a control system. The control flow of the Base part is as follows. First, a user is detected by the Kinect V2 sensor, and a target vector command is sent from the $\mathrm{PC}$ to the microcomputer according to the information received from the sensor. The microcomputer calculates speed and direction from each parameter and control the motors via the servo controller.

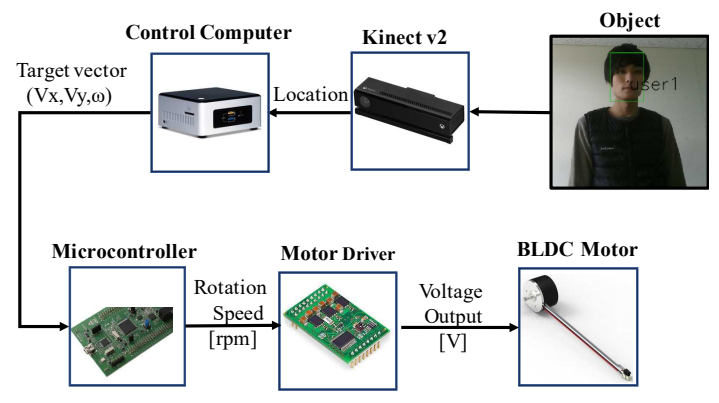

Fig. 3. Control diagram

\section{Motion Model of the Moving Device}

The mecanum wheel adopted for the Base part is covered with a barrel whose surface is inclined at 45 degree with respect to the axle. By controlling the rotation direction of each wheel as shown in Figure 4, omnidirectional movement is realized by a combination of rotation of the wheel and movement of the barrel on the circumference.

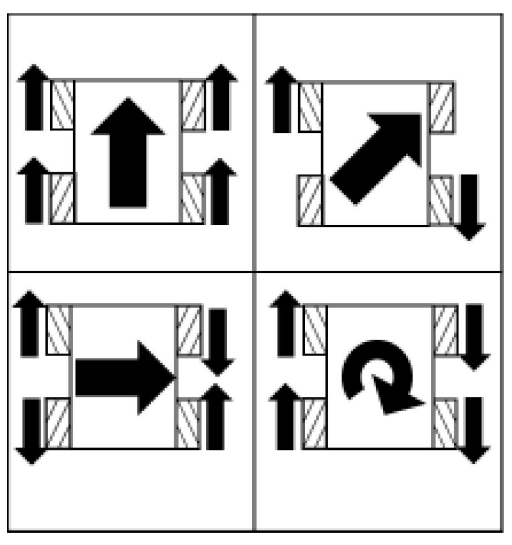

Fig. 4. Various combination of wheel rotation

Let $\mathrm{V}_{0}$ be the vector according to the angular velocity. Let $V_{w}$ be a velocity vector in rectangular coordinates. In equation (3), "I" represents the distance from the center of gravity to the lateral edge, and "L" represents the distance to the longitudinal edge.

$$
\begin{gathered}
V_{w}=\left[V_{1 w} V_{2 w} V_{3 w} V_{4 w}\right]^{T} \in R^{4 \times 1} \\
V_{0}=\left[V_{x} V_{y} \omega_{z}\right]^{T} \in R^{3 \times 1} \\
J_{0}=\left[\begin{array}{ccc}
1 & -1 & -(l+L) \\
1 & 1 & (l+L) \\
1 & 1 & -(L+l) \\
1 & -1 & (L+l)
\end{array}\right]
\end{gathered}
$$


$V_{W}$ can be expressed by using the equation (2), (3).

$$
V_{w}=J_{0} \cdot V_{0}
$$

Here, the speed control of the moving device gives the vector $\left(V_{x}, V_{y}, \omega_{z}\right)$ as input to the microcomputer and controls the speed $V_{w}$ of each wheel by PWM output.

\section{System Flow of the Movement Control}

Figure 5 shows the flowchart of the movement control of the Base part. In the movement control, base part is controlled so as to keep a specific position according to the sensor value. Specifically, the distance and angle are calculated, and the robot approaches the user until the distance of the threshold.

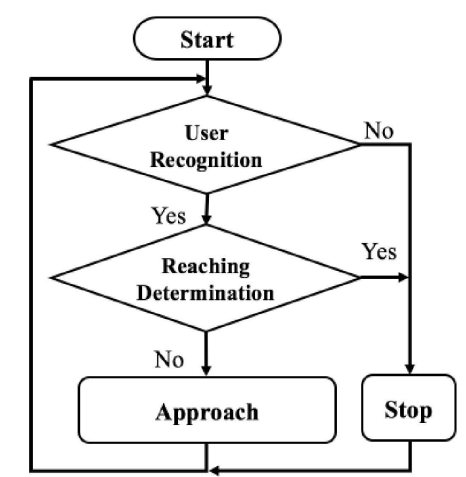

Fig. 5. Flow chart of location control

\section{Measurement of the personal space}

As I said in chapter 2, People update, control, and adjust their personal spaces continuously. Therefore, psychological strains between human to robot could be measured by physical distances.

In this time, the personal space between robot and subject were measured using the following procedures.

(i) The robot approached at each velocity $(0.2,0.3,0.4$, $0.5,0.6[\mathrm{~m} / \mathrm{s}])$ to a subject who was standing

(ii) The subject raised his hands as a signal if he felt uncomfortable (too close to the robot).

(iii) The distance was measured by a sensor installed at the front of the robot and these procedures were repeated for the 10 subjects.

The measurement result is depicted in Figure 6.
As the velocity increased, the standard deviation tended to be higher. However, there was no significant relationship between the velocity and the distance. The average distance was almost same as any velocity.

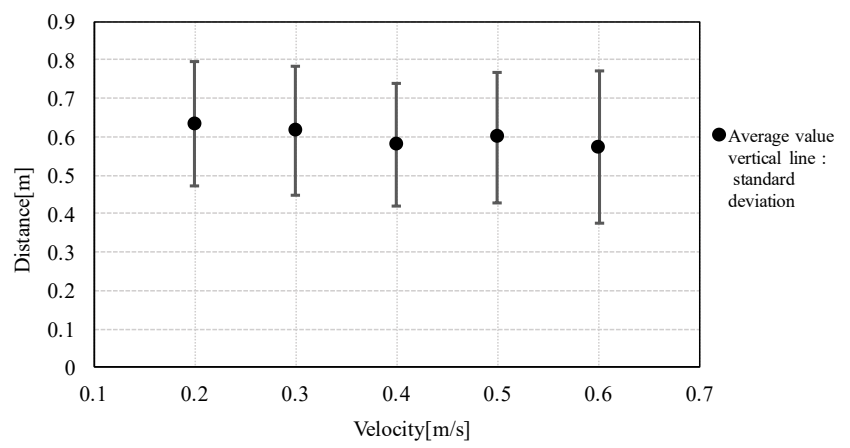

Fig. 6. Measurement result

\section{Evaluation of the personal distance}

Based on the measurement values obtained from the previous experiment, the evaluation experiments were conducted. The moving speed was controlled within the range of $0.0 \sim 0.3[\mathrm{~m} / \mathrm{s}]$ so that the boundary of the personal space was kept about $0.6 \mathrm{~m}$, which was the average distance of the previous experiment.

The evaluation experiment was conducted as follows.

(i) A subject sat on the chair on casters in front of the robot.

(ii) The subject moved freely in the specified range for a minute.

(iii) The robot kept the distance as shown in Figure 7.

(iv) After the experiment, the subject answered the questionnaire of the impression about the robot's approach. Repeat for the 12 subjects.

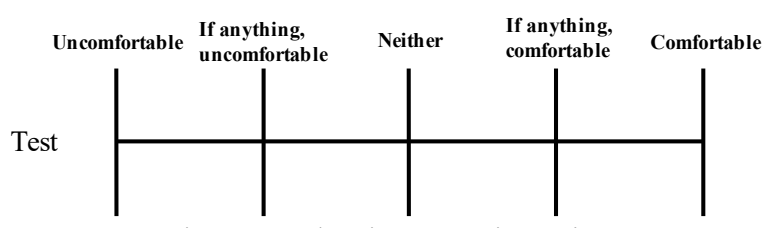

Fig. 7. Evaluation questionnaire

The result of the evaluation experiment is depicted in Figure8. Each result of the questionnaire was quantified as shown in Table 2. 


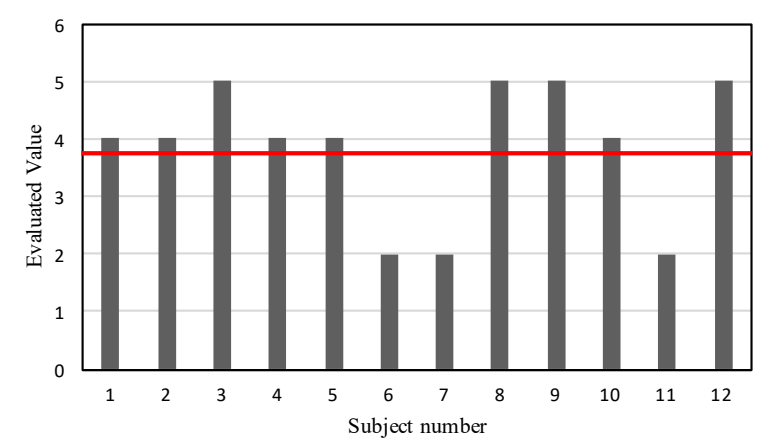

Fig. 8. Evaluation result

Table. 2. Conversion table for the result

\begin{tabular}{|l|c|c|c|c|c|}
\hline & $\begin{array}{c}\text { Uncom- } \\
\text { fortable }\end{array}$ & ----- & $\begin{array}{c}\text { Nei- } \\
\text { ther }\end{array}$ & $\begin{array}{c}----- \\
\text { Com- } \\
\text { fortable }\end{array}$ \\
\hline $\begin{array}{l}\text { Evaluated } \\
\text { value }\end{array}$ & 1 & 2 & 3 & 4 & 5 \\
\hline
\end{tabular}

Table.3.Average and standard deviation of the result

\begin{tabular}{|l|c|}
\hline average & 3.8 \\
\hline Standard deviation & 1.1 \\
\hline
\end{tabular}

The average score is shown by a red horizontal line. The average score was 3.8 and close to "comfortable".

\section{Conclusion and Future work}

It is considered that the velocity range and the distance could be adapted to realize the appropriate approach to most of the users. It means that the distance can be index of initial distance between robot and users. However, to optimize the personal space for each users, we need to create the database to store the information.

\section{References}

[1] S.Yumitori, Overview of Robot R\&D in NEDO

[2] K.Buhardwaj, Professional Communication

[3] E.T. Hall, The Hidden Dimension (Vol. 609. Garden City, NY: Doubleday, 1966).

[4] J.leroy, M.mancas and B.gossel, Personal Space Augmented Reality Tool 